\title{
Same-day discharge after brain tumor resection: a prospective pilot study
}

\author{
Frederic A. Vallejo ${ }^{1}$ (1) - Daniel G. Eichberg ${ }^{1}$. Alexis A. Morell ${ }^{1}$. Ashish H. Shah ${ }^{1}$ - Long Di $^{1} \cdot$ Katherine Berry $^{1}$. \\ Evan Luther ${ }^{1} \cdot$ Victor M Lu ${ }^{1} \cdot$ Nitesh V. Patel ${ }^{1} \cdot$ Michael E. Ivan ${ }^{1} \cdot$ Ricardo J. Komotar ${ }^{1}$
}

Received: 11 January 2022 / Accepted: 14 February 2022 / Published online: 22 February 2022

(c) The Author(s), under exclusive licence to Springer Science+Business Media, LLC, part of Springer Nature 2022

\begin{abstract}
Purpose Outpatient brain surgery has many advantages for the psychological and physical wellbeing of patients, as well as reduced costs to the health care system. Compared with inpatient admissions, same day discharges reduce patient exposure to nosocomial infection, thromboembolic complications, and medical error. We aim to establish a prospectively collected quality outcomes database to examine the outcomes of patients that undergo brain tumor resection and are discharged home the same day as surgery.

Methods We have established a prospectively collected quality outcomes database to examine the outcomes of all patients that underwent brain tumor resection by a single neurosurgeon (R.J.K) at our institution from August 2020 to August 2021 and were discharged home the same day as surgery.

Results Over the one-year period this study was conducted, 37 of 334 patients met inclusion criteria for the outpatient protocol. Thirty-two patients were discharged on the same day as surgery. Five patients (14\%) were considered eligible for outpatient surgery but were ultimately admitted to the hospital postoperatively and were discharged after an overnight observation. No postoperative complications were noted at two-week postoperative follow-up.

Conclusion In select patients undergoing brain tumor surgery, same day discharge should be considered. Establishing a multidisciplinary team of physicians, nurses, radiologists, and physical therapists is critical to achieving this aim. Physicians should have a low threshold to admit a patient with concerning exam findings, complications, or complicated past medical history. Once discharged, open communication with the patient and their family is critical to detect complications that should trigger rehospitalization and intervention.
\end{abstract}

Keywords Brain tumor $\cdot$ Neuro-oncology $\cdot$ Neurosurgical outcomes $\cdot$ Outpatient craniotomy $\cdot$ Same-day discharge

\section{Introduction}

Cranial neurosurgery confers psychological stress due to the perceived invasiveness of the procedures, as well as the stress of being in the hospital rather than at home on one's preferred surroundings [1]. Hospitalization also confers risks such as hospital acquired infections, medical errors, and deep tissue injuries such as pressure ulcerations. Because of the risks associated with long postsurgical inpatient hospitalizations, some neurosurgeons have challenged the standard

Frederic A. Vallejo

Fav8@med.miami.edu

1 Department of Neurosurgery, University of Miami Miller School of Medicine Lois Pope Life Center, 1095 14th Terrace, Miami, FL 33136, USA practice of admitting all postoperative patients for at least one-night following craniotomy for observation in order to attempt to progressively reduce the time patients must spend inpatient without sacrificing safety.

Previous retrospective studies on same-day discharge have shown this practice to be safe, effective, and beneficial for certain patients [2-6]. These findings, led by the University of Toronto, assert that outpatient craniotomy is safe in neuro-oncology patients who had supra-tentorial tumors resected or biopsied. Following a similar approach, we conducted the first prospective study in the United States to establish a quality outcomes database examining the outcomes of patients that undergo brain tumor surgery and are discharged home the same day as surgery. We posit that, with proper patient selection, same-day discharge is safe and can improve patient flow through our neurosurgical service 
due to more effective allocation of postoperative inpatient resources. Here, we report the results of a pilot study prospectively evaluating outpatient brain tumor resection in a series of 37 selected patients treated over one year at our institution.

\section{Methods}

\section{Study population}

The University of Miami institutional review board approved the present study (protocol \#20201078). A prospective quality intervention study including patients who underwent outpatient surgery for brain tumors by a single neurosurgeon (R.J.K) at the University of Miami from August 2020 to August 2021 was performed. Complete demographic, anatomic, and clinical data were collected prospectively for each patient from their inpatient admission, clinic followup, and any readmission records. Hospital readmissions were classified as very early (1-3 days after the surgery) and early (4-30 days after the surgery). The electronic medical records, operative notes, radiographic films, and final pathology reports were also reviewed. This study was adherent to the Strengthening the Reporting of Observational Studies in Epidemiology (STROBE) criteria.

\section{Inclusion criteria for outpatient surgery}

We considered eligible for outpatient surgery all patients between 16 and 85 years of age, with an American Society of Anesthesiologists (ASA Score) physical status classification system $\leq 4$ and a Karnofsky Performance Score $\geq 70$, who were undergoing craniotomy for suspected supratentorial brain tumor at the University of Miami Hospital. Additional inclusion criteria included tumors with a maximum diameter of $4 \mathrm{~cm}$, surgeries with predicted blood loss of less than $300 \mathrm{ml}$, and a predicted maximum surgery length of less than $3 \mathrm{~h}$. Both surgeries under general endotracheal anesthesia and awake surgeries utilizing monitored anesthesia care were considered eligible. All eligible patients were reviewed by the attending neurosurgeon to determine if a patient was expected to be ready for safe discharge home the same day as surgery (Fig. 1). Attending neurosurgeons remained able to modify their decision at any moment and admit the patient if it was considered necessary by clinical judgement. Patients must give informed consent to be included in the study. This study was conducted in accordance with IRB requirements with the intention of collecting prospective data for this pilot study.

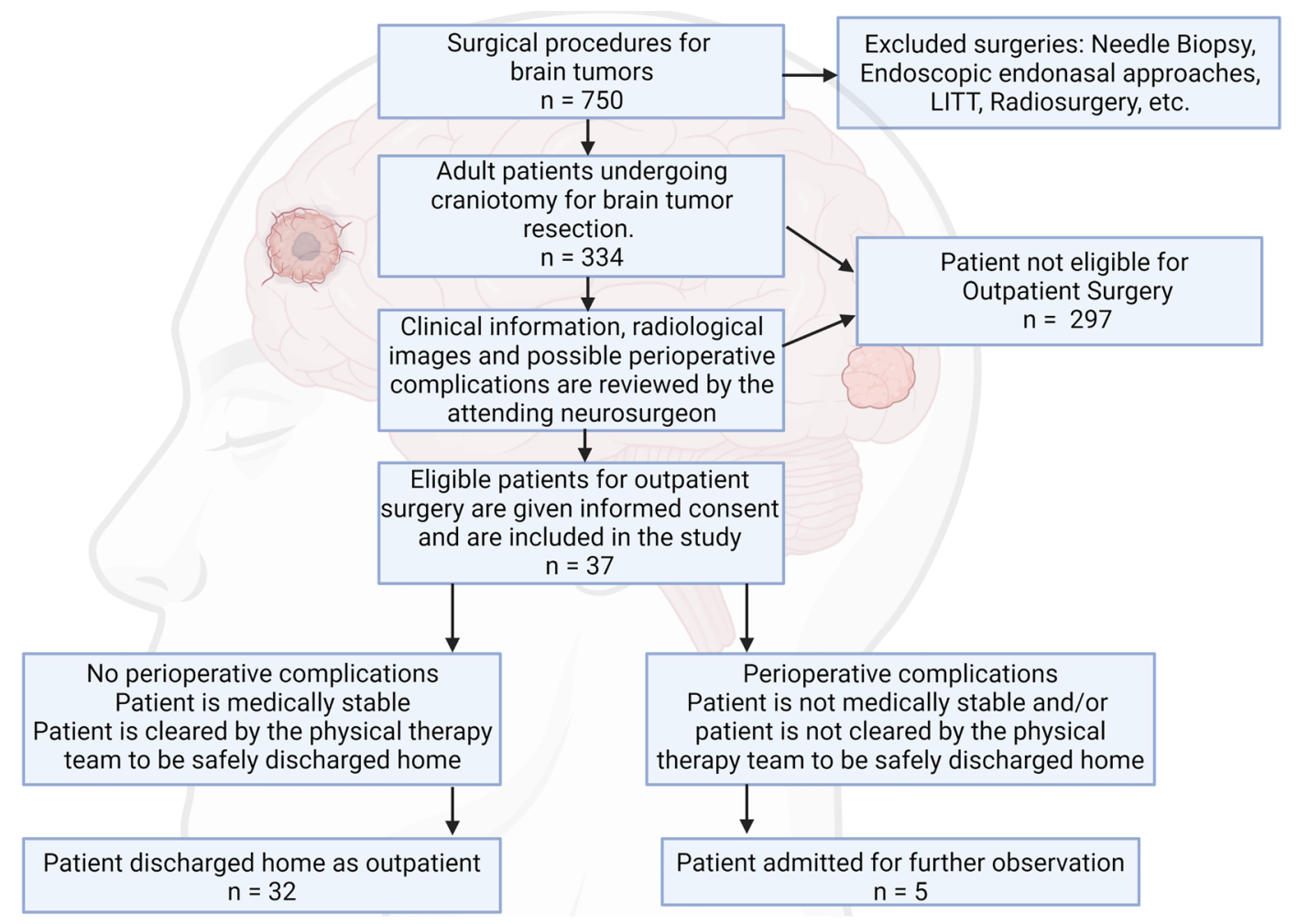

Fig. 1 Outpatient surgery protocol and patients included from August 2020 to August 2021 


\section{Exclusion criteria}

Individuals with lesions carrying a higher risk of surgical morbidity as infratentorial or intraventricular tumors were not included in the outpatient protocol. Also, patients with a Body Mass Index (BMI) of more than $35 \mathrm{~kg} / \mathrm{m}^{2}$, limited home support, pituitary or skull base tumors, or requiring CSF diversion were not considered eligible for outpatient surgery. Surgeries were planned to start prior to 8:30 AM in order to qualify for same-day discharge.

\section{Admission and pre-op clearance}

Patients were admitted the evening before surgery to get preop clearance and a contrast-enhanced fiducial MRI.

\section{Postoperative monitoring}

After surgery, patients who underwent tumor resection were observed for $3 \mathrm{~h}$ in the Post-anesthesia Care Unit (PACU), followed by a contrast-enhanced MRI. Once the MRI was acquired, the patient was monitored in the NeuroICU for an additional $3 \mathrm{~h}$. In all patients, the minimum observation period was $6 \mathrm{~h}$ after surgery.

\section{Discharge and follow-up}

Patients were deemed safe for discharge when they were hemodynamically stable, had been cleared by physical and occupational therapy, had completed postoperative imaging without any concerning neurosurgical findings, and had passed a complete neurological assessment. To be medically clear for discharge, patients had to be afebrile, have no issue with their surgical incision, and adequate pain-management had to be obtained. The goal of physical and occupational therapy was to ensure patients were able to eat, drink, and walk without assistance prior to discharge.

The attending neurosurgeon determined if a patient was safe to discharge home on the same day as surgery, or if the patient would be admitted to the hospital. If there was any concern about the patient's medical status, or if the physical therapy team did not clear the patient for safe discharge home, the patient was admitted to the hospital. All patients voluntarily consented for this study and the priority of the team was to ensure each patient felt safe and secure with the discharge plan. As such, if a patient were to feel more secure leaving after overnight inpatient-monitoring, this decision would be reached collaboratively putting the patient's well-being first. Patients were seen two weeks postoperatively in outpatient clinic for routine follow-up (Fig. 2).

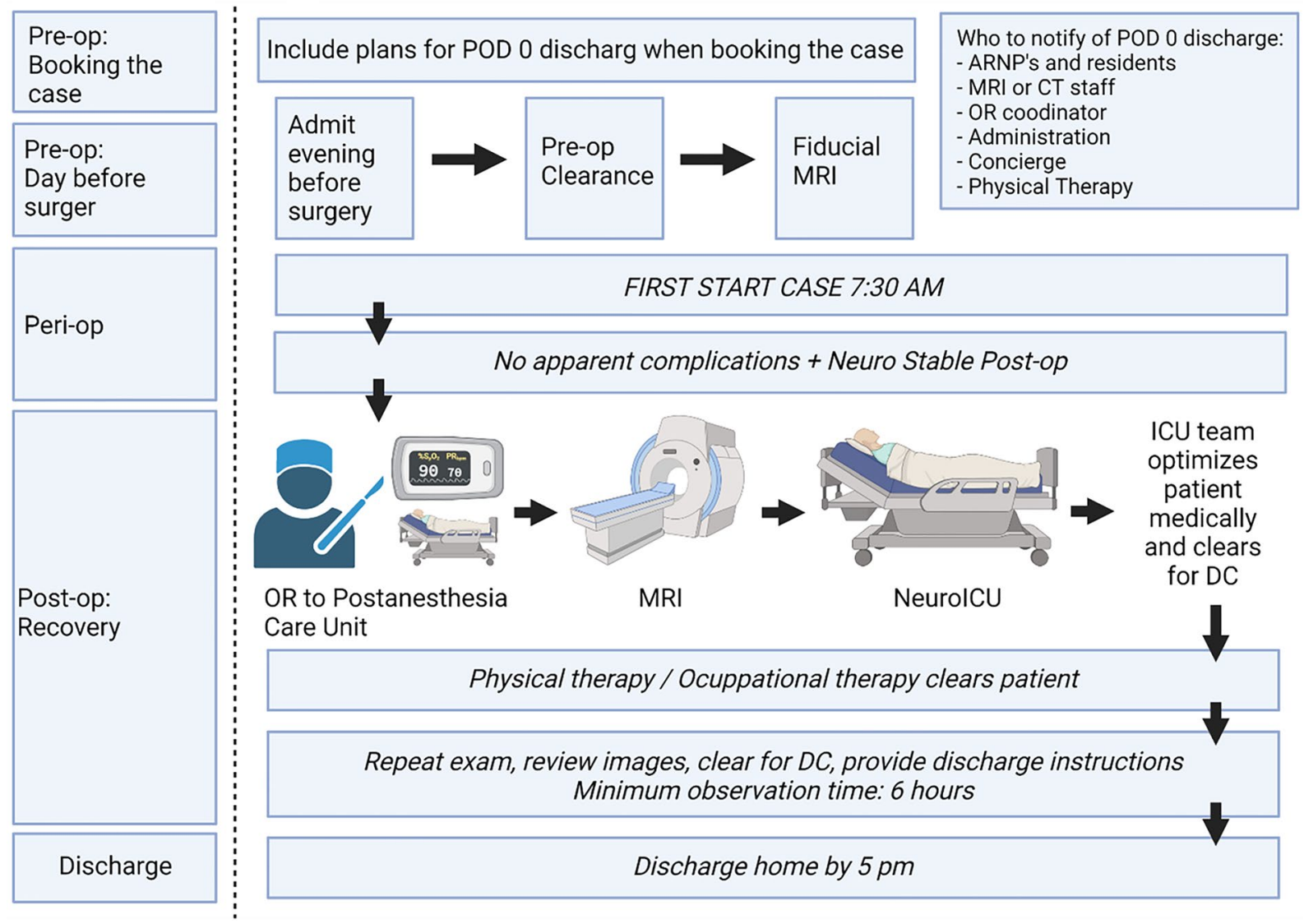

Fig. 2 Flow Chart describing outpatient management in brain tumor resection and brain tumor biopsy 


\section{Statistical analysis}

All statistical analysis was performed with IBM SPSS v26.0 (IBM, Armonk, NY) at an alpha value of 0.05. A qualitative and quantitative comparison between discharged and admitted patients was performed. Ordinal variables were compared using a Mann-Whitney U test with two-sided and one-sided comparisons and exact significance values corrected for ties reported. Nominal variables were compared using a Fisher Exact Test. Lastly, continuous variables were compared using independent samples t-test. Figures were created with Biorender.com.

\section{Results}

Thirty-seven consecutive brain tumor patients who had surgical interventions over a one year period between August of 2020 and August of 2021 were included in this study (Table 1). The median patient-age was 52 years with $32 \%$ (12/37) of patients being male and 68\% (25/37) being female. Frontal-lobe lesions accounted for 49\% (18/37) of the surgeries, $27 \%$ (10/37) located in the temporal region, $14 \%(5 / 37)$ in the parietal region, 5\% (2/37) in the occipital region, and multiple locations in 5\% (2/37). Additionally, $70 \%(26 / 37)$ of tumors were primary lesions whereas $30 \%$ $(11 / 37)$ tumors exhibited pathology indicative of metastatic disease. All tumors operated on in this study were supratentorial lesions and all operations were performed without complications. Anesthesia was administered as part of an awake procedure in seven patients (19\%), with the rest receiving standard general anesthesia $(81 \%)$. The average BMI across all patients was $25.80 \mathrm{~kg} / \mathrm{m}^{2}(17.4-32.24)$, while ASA score was 1 in one patient (3\%), 2 in fifteen patients (41\%), 3 in twenty-one (56\%). Lastly, the median tumor volume resected across the thirty-seven patient cohort was $3.48 \mathrm{~mL}$, with a mean tumor volume of $4.58 \mathrm{~mL}$ (0.033-23.65) (Fig. 3).

Of the 37 patients who were included in the study, 32 (86\%) patients were discharged on the same day of surgery (Table 2). Five of the thirty-seven (14\%) of the patients who underwent open tumor resection were not deemed to be eligible for same day discharge. One of the patients remained in the hospital an additional night due to lack of social support. The remaining four patients had to be admitted for heightened postoperative monitoring. All four patients admitted were successfully discharged $24 \mathrm{~h}$ after the surgery. The rationale behind further inpatient monitoring in these cases was because one patient with known epilepsy had presented with a generalized seizure prior to surgery, one patient with post-operative headache, unsteadiness in another patient, and severe postoperative abdominal discomfort in the last.
Table 1 Characteristics of the patients included

\begin{tabular}{|c|c|}
\hline Variable & No. $(\%)$ \\
\hline Total no. of patients & $37(100 \%)$ \\
\hline Female & $25(68 \%)$ \\
\hline Male & $12(32 \%)$ \\
\hline \multicolumn{2}{|l|}{ Median age in years, range } \\
\hline All patients & $52,18-81$ \\
\hline Female & $53,21-81$ \\
\hline Male & $49,18-75$ \\
\hline Diabetes & $3(8 \%)$ \\
\hline \multicolumn{2}{|l|}{ Tumor location } \\
\hline Frontal & $18(49 \%)$ \\
\hline Temporal & $10(27 \%)$ \\
\hline Parietal & $5(14 \%)$ \\
\hline Occipital & $2(5 \%)$ \\
\hline Multiple & $2(5 \%)$ \\
\hline \multicolumn{2}{|l|}{ Diagnosis } \\
\hline Meningioma & $14(38 \%)$ \\
\hline Metastatic disease & $11(30 \%)$ \\
\hline Cavernoma & $2(5 \%)$ \\
\hline Glioblastoma & $1(3 \%)$ \\
\hline Osteoblastoma & $1(3 \%)$ \\
\hline Langerhans Cell Histiocytosis & $1(3 \%)$ \\
\hline Benign Hemangioma & $1(3 \%)$ \\
\hline Inflammatory Process & $1(3 \%)$ \\
\hline SMART & $1(3 \%)$ \\
\hline Radiation necrosis & $1(3 \%)$ \\
\hline Grade III Glioma & $1(3 \%)$ \\
\hline Lipoma & $1(3 \%)$ \\
\hline Cortical Dysplasia & $1(3 \%)$ \\
\hline \multicolumn{2}{|l|}{ Anesthesia } \\
\hline General & $30(81 \%)$ \\
\hline Awake & $7(19 \%)$ \\
\hline \multicolumn{2}{|l|}{ BMI in $\mathrm{kg} / \mathrm{m}^{2}$} \\
\hline Median & 24.82 \\
\hline Range & $17.4-32.24$ \\
\hline \multicolumn{2}{|l|}{ ASA Score } \\
\hline 1 & $1(3 \%)$ \\
\hline 2 & $15(41 \%)$ \\
\hline 3 & $21(56 \%)$ \\
\hline \multicolumn{2}{|l|}{ Karnofsky Performance Score } \\
\hline Median & 90 \\
\hline Range & $70-100$ \\
\hline \multicolumn{2}{|l|}{ Resected tumor volume in $\mathrm{mL}$} \\
\hline Mean & 4.58 \\
\hline Median & 3.48 \\
\hline Range & $.033-23.65$ \\
\hline
\end{tabular}

Very early hospital readmissions (1-3 days after the surgery) were not seen in any of the patients. Early readmissions (4-30 days after the surgery) occurred in 4 patients. 


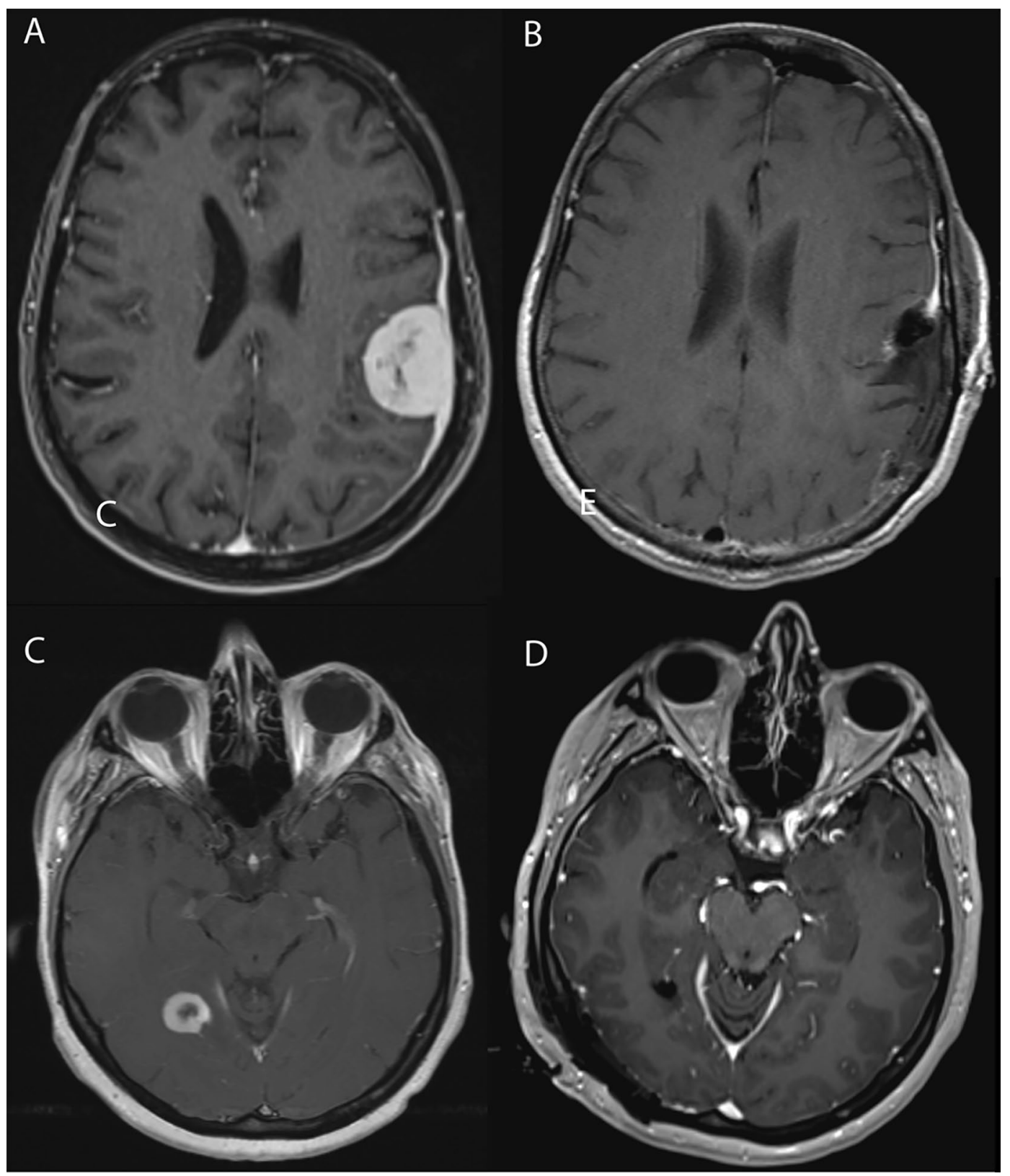

Fig. 3 Pre-operative and post-operative images of patients who underwent surgical resection of a brain tumor. The first patient (A, B) is a 73 year old female who underwent gross total resection of a WHO Grade 1 meningioma, measuring $3 \times 3.4 \times 3.4 \mathrm{~cm}$. The second patient $(\mathbf{C}, \mathbf{D})$ is a 45 y.o. female with a history of breast cancer pre-

The cause of the readmission was related to the perioperative course in one patient, as he presented with hypotension nine days after surgery and was diagnosed with acute adrenal insufficiency. For the other three patients, the cause of the readmission was not related to the surgery. One readmission was due to seizures in another patient who was epileptic prior to surgery. Another was due to abdominal pain and constipation. The final readmission was due to back pain in senting with a solitary metastasis, completing her adjuvant treatment with chemotherapy and stereotactic radiosurgery after gross total resection. The patients were medically stable and were discharged home after being cleared using our protocol (Fig. 2)

the setting of spinal metastases. All patients readmitted were discharged successfully after adequate care.

\section{Analysis of quantitative variables}

A comparison of quantitative variables of age, body-mass index (BMI), and pre-operative KPS and ASA between patients discharged on the same day versus patients later admitted postoperatively was performed. Age was the only 
Table 2 Outcomes after surgery

\begin{tabular}{ll}
\hline & No. $(\%)$ \\
\hline Discharged as outpatient & $32(86 \%)$ \\
Discharged on postoperative day 1 & $5(14 \%)$ \\
Hospital readmissions (1-30 days postop.) & \\
Immediate (1-3 days postop.) & $1(0 \%)$ \\
Early (4-30 days postop.) & $4(100 \%)$ \\
\hline
\end{tabular}

variable that was statistically significantly different between patients discharged on POD0 and patients later admitted. Patients that were discharged on the same day were significantly younger that patients later admitted (50.2 vs. 66.2 yo, $\mathrm{p}=0.042$ ). There was no significant difference in BMI or preoperative KPS and ASA between same-day discharge and later admission $(p=0.643, p=0.111, p=0.259)$.

\section{Analysis of qualitative variables}

On Fisher Exact Test, emergency room (ER) visit and consultation was trended towards an association with increased rates of hospital admission $(p=0.080)$. Forty percent of patients with ER visit were later admitted whereas only two percent of patients that were discharged on POD0 had a prior ER visit. There was no significant association on Fisher Exact Test between same day discharge and gender, history of diabetes or sleep apnea, type of intraoperative anesthesia, or incidence of post-operative neurologic or systemic complications $(\mathrm{p}>0.05)$. Summary statistics may be reviewed in Table 3 .

\section{Discussion}

We previously published our institutional experience with discharging patients on postoperative day one (POD1) after craniotomy for brain tumors [7]. We compared complication and readmission rates between patients discharged on POD1 and those with longer stays, as well as predictive factors of successful POD1 discharge. Of patients with $>1$ day of hospital stay, $4.4 \%$ experienced a deep-vein thrombosis (DVT) or pulmonary embolism (PE) and $2.7 \%$ experienced urinary tract infection (UTI). In contrast, patients discharged on POD1 experienced no DVTs, PEs, or UTIs and had significantly lower 30-day rates of readmission to the hospital. Patients discharged on POD1 had significantly better scores on both the Karnofsky performance scale and the modified Rankin scale ( $\mathrm{P}<0.0001$ for both). Multivariate regression analysis revealed several patient, tumor, and operative factors which were independent predictors of successful discharge on POD1. These factors included: lower modified frailty index score, male sex, right sided tumors, supratentorial tumors, smaller tumor diameter, and awake surgery.
Conversely, patients with tumors located in the posterior fossa, the use of general endotracheal anesthesia, and cerebrospinal fluid drain placement were associated with longer inpatient stays.

Given our consistent positive patient-experience with POD1 discharge, we aimed to extend this opportunity to same-day-discharge (POD0) and prospectively confirm its feasibility in our brain tumor population. Outpatient craniotomy has not been shown to increase morbidity in patients who received either monitored or general anesthesia for their operations $[6,8,9]$. Although spending a night in the hospital before the procedure is contrary to the concept of ambulatory surgery, there is data showing that patients who are admitted on the same day as surgery have a higher stress level (measured by preoperative heart rate and skin conductance), compared to patients admitted the evening prior [10]. Having additional time available before surgery for preparation at a slower pace may have a calming effect on patients [10]. Patients discharged on POD0 have reported high levels of satisfaction, as avoiding an overnight stay in the hospital may lessen some of the psychological stress accompanying brain tumor resection [11]. Outpatient tumor resection may be beneficial on three fronts: optimizing bed-usage for patients who need more aggressive surveillance, decreasing hospital-related complications, thus preventing avoidable postoperative readmissions and driving down costs, and reducing potential cancellations due to a paucity of available beds. Additionally, high-volume surgical centers with same-day imaging capabilities may particularly benefit from implementing same-day discharge at their institutions when there are limited ICU beds available during SARS-CoV-2 or influenza surges.

Whereas same-day discharge for neuro-endovascular treatment of unruptured aneurysms and both minimally invasive cervical and lumbar spinal surgery is commonplace in modern practice, adopting regular outpatient brain surgery has been met with more resistance and apprehension [12-15]. Other surgical procedures across multiple disciplines, which historically admitted patients after the operation, have also found that same-day discharge is a safe and viable option $[16,17]$. Regarding awake craniotomy, surgeons have been able to greatly reduce complications by avoiding eloquent regions with intra-operative stimulation under local, less aggressive sedation [18, 19]. General anesthesia does not preclude the possibility of same-day discharge and patients should be considered on a case-by-case basis [6].

Safe outpatient brain surgery is heavily dependent on appropriate patient selection. Between August 2020 and August 2021, the senior author (R.J.K.) performed 334 craniotomies for brain tumors. From that population, only 37 patients were eligible for outpatient management, showing 
Table 3 Summary statistics for patient cohort

\begin{tabular}{|c|c|c|c|}
\hline Variable & Discharged $(\mu \pm$ S.D. $)$ & Admitted $(\mu \pm$ S.D. $)$ & P-value \\
\hline Age & $50.2 \pm 16.3$ & $66.2 \pm 9.9$ & .042 \\
\hline Variable & $\begin{array}{l}\text { Discharged } \\
\mathrm{n}(\%)\end{array}$ & $\begin{array}{l}\text { Admitted } \\
\mathrm{n}(\%)\end{array}$ & P-value \\
\hline Gender & & & 1.000 \\
\hline Female & $22(68.8)$ & $3(60)$ & \\
\hline Male & $10(31.2)$ & $2(40)$ & \\
\hline Diabetes & & & .362 \\
\hline No & $30(93.8)$ & $4(80)$ & \\
\hline Yes & $2(6.2)$ & $1(20)$ & \\
\hline Anesthesia & & & .560 \\
\hline Awake & $7(21.9)$ & $0(0)$ & \\
\hline General & $25(78.1)$ & $5(100)$ & \\
\hline Sleep арпеа & & & .362 \\
\hline No & $30(93.8)$ & $4(80)$ & \\
\hline Yes & $2(6.2)$ & $1(20)$ & \\
\hline ER visit & & & .080 \\
\hline No & $30(93.8)$ & $3(60)$ & \\
\hline Yes & $2(6.2)$ & $2(40)$ & \\
\hline Neurologic complications & & & - \\
\hline No & $32(100)$ & $5(100)$ & \\
\hline Yes & $0(0)$ & $0(0)$ & \\
\hline Systemic complications & & & 1.000 \\
\hline No & $31(96.9)$ & $5(100)$ & \\
\hline Yes & $1(3.1)$ & $0(0)$ & \\
\hline Variable & Discharged (mean rank) & Admitted (mean rank) & P-value \\
\hline KPS & 20 & 12.4 & .111 \\
\hline ASA & 18.3 & 23.4 & .259 \\
\hline
\end{tabular}

that even in a high-volume brain tumor practice nearly $90 \%$ of the patients still need to be admitted to the hospital.

In our series, $86 \%(32 / 37)$ of the patients were successfully discharged on POD0 with a 30-day surgery-related readmission of $3 \%(1 / 37)$ and no complications. In their series, the University of Toronto published similar results, ranging between $86-94 \%$ of successful discharge rate, with $5-11 \%$ of complications [2, 3, 6, 20, 21].

Although complications are rare, they tend to present within hours of the operation rather than days. For example, intracranial neurosurgical procedures have been shown to typically elicit postoperative hematoma within $6 \mathrm{~h}$ of surgery [22]. In a recent study of 200 consecutive elective craniotomy patients, $4 \mathrm{~h}$ of postoperative monitoring in the PACU was found to be a sufficient amount of time before safely moving the patient to a neurosurgical step-down unit [23].

Outpatient brain surgery in select cases is not only safe, but also can increase patient quality of life. Reducing length of hospital stay after resection enables patients to get back to their homes and families, allowing them to return to a sense of normalcy more quickly. Patient's perception of their individual healing processes heavily depends on "being cared for, being comfortable, and experiencing something familiar or like home," which may be part of the reason why outpatient brain surgery has very positive feedback and high levels of satisfaction [11, 24]. Oftentimes, patients are confronted with psychological and physical stress associated not only with their neuro-oncological pathology, but also due to the financial burden that often accompanies highly specialized surgical interventions.

The fear of legal repercussions after rare complications secondary to early discharge both in the United States and around the world may be one factor deterring cranial surgeons from more openly adopting outpatient craniotomy in their practices as they threaten to undo the financial benefits that incentivize same-day discharge on an institutional level [25]. This emphasizes the critical role attending neurosurgeons play in selecting appropriate patients for which they believe same-day discharge will increase patient outcome 
and quality of care. On the contrary, the increase in costeffectiveness and limitation of resource expenditures may actually directly enable neurosurgical care previously not possible in underserved parts of the world [26]. Given sufficient resources, transportation, and access to appropriate medication, certain areas of the developing world may entertain surgical intervention for eligible candidates which they may not previously have been able to operate on given cost and resource restraints. This being said, physicians in the United States and other countries should have a low threshold to admit a patient with concerning exam findings, complications, or complicated past medical history.

\section{Limitations}

We identified age as a variable positively associated with later hospital admission. Patients who visited the ER for consultation trended towards a greater likelihood of postoperative admission but this was not statistically significant. In regards to these findings, our study was of only moderate sample size and may have been underpowered to identify other significant associations should they exist. Ultimately, we believe the data garnered from this preliminary proofof-principle study warrants further investigation in larger, prospective cohort trials.

\section{Conclusions}

With appropriate selection and postoperative monitoring, same day discharge can be considered a safe and feasible option for certain craniotomy cases. Outpatient brain surgery has been shown to be a safe and effective method for postoperative management while optimizing flow through the neurosurgical service by allocating resources to where they are most needed. Establishment of clear protocols for patient selection and patient education is central to the success of same-day discharge for brain tumor resection. We hope that our experience and prospective database enables other institutions to explore the feasibility of offering outpatient brain surgery for their neuro-oncology patients.

Funding The authors declare that no funds, grants, or other support were received during the preparation of this manuscript.

Data availability No data sets were generated or analyzed during the current study.

\section{Declarations}

Conflict of interest The authors have no relevant financial or non-financial interests to disclose.

Ethical approval This study was performed in line with the principles of the Declaration of Helsinki. IRB approval was granted by the Ethics Committee of University of Miami (protocol \#20201078).

Consent to participate Informed consent was obtained from all individual participants included in the study.

\section{References}

1. Palese A, Cecconi M, Moreale R, Skrap M (2012) Pre-operative stress, anxiety, depression and coping strategies adopted by patients experiencing their first or recurrent brain neoplasm: an explorative study. Stress Health 28:416-425. https://doi.org/10. 1002/smi.2472

2. Bernstein M (2001) Outpatient craniotomy for brain tumor: a pilot feasibility study in 46 patients. Can J Neurol Sci 28:120-124. https://doi.org/10.1017/s0317167100052781

3. Boulton M, Bernstein M (2008) Outpatient brain tumor surgery: innovation in surgical neurooncology. J Neurosurg 108:649-654. https://doi.org/10.3171/jns/2008/108/4/0649

4. Bhardwaj RD, Bernstein M (2002) Prospective feasibility study of outpatient stereotactic brain lesion biopsy. Neurosurgery 51:358361 (discussion 361-354)

5. Grundy PL, Weidmann C, Bernstein M (2008) Day-case neurosurgery for brain tumours: the early United Kingdom experience. Br J Neurosurg 22:360-367. https://doi.org/10.1080/0268869080 1961858

6. Au K, Bharadwaj S, Venkatraghavan L, Bernstein M (2016) Outpatient brain tumor craniotomy under general anesthesia. J Neurosurg 125:1130-1135. https://doi.org/10.3171/2015.11.Jns152151

7. Richardson AM, McCarthy DJ, Sandhu J, Mayrand R, Guerrero C, Rosenberg C, Gernsback JE, Komotar R, Ivan M (2019) Predictors of successful discharge of patients on postoperative day 1 after craniotomy for brain tumor. World Neurosurg 126:e869e877. https://doi.org/10.1016/j.wneu.2019.03.004

8. Purzner T, Purzner J, Massicotte EM, Bernstein M (2011) Outpatient brain tumor surgery and spinal decompression: a prospective study of 1003 patients. Neurosurgery 69:119-126. https://doi.org/ 10.1227/NEU.0b013e318215a270 (discussion 126-117)

9. Sheshadri V, Venkatraghavan L, Manninen P, Bernstein M (2018) Anesthesia for same day discharge after craniotomy: review of a single center experience. J Neurosurg Anesthesiol 30:299-304. https://doi.org/10.1097/ana.0000000000000483

10. Wetsch WA, Pircher I, Lederer W, Kinzl JF, Traweger C, HeinzErian P, Benzer A (2009) Preoperative stress and anxiety in daycare patients and inpatients undergoing fast-track surgery. Br J Anaesth 103:199-205. https://doi.org/10.1093/bja/aep136

11. Khu KJ, Doglietto F, Radovanovic I, Taleb F, Mendelsohn D, Zadeh G, Bernstein M (2010) Patients' perceptions of awake and outpatient craniotomy for brain tumor: a qualitative study. J Neurosurg 112:1056-1060. https://doi.org/10.3171/2009.6.Jns09716

12. Goettel N, Chui J, Venkatraghavan L, Tymianski M, Manninen PH (2014) Day surgery craniotomy for unruptured cerebral aneurysms: a single center experience. J Neurosurg Anesthesiol 26:60-64. https://doi.org/10.1097/ANA.0b013e3182991d8b

13. Zahrawi $\mathrm{F}$ (1994) Microlumbar discectomy. Is it safe as an outpatient procedure? Spine (Phila Pa 1976) 19:1070-1074 
14. McGirt MJ, Godil SS, Asher AL, Parker SL, Devin CJ (2015) Quality analysis of anterior cervical discectomy and fusion in the outpatient versus inpatient setting: analysis of 7288 patients from the NSQIP database. Neurosurg Focus 39:E9. https://doi.org/10. 3171/2015.9.Focus 15335

15. Pugely AJ, Martin CT, Gao Y, Mendoza-Lattes SA (2013) Outpatient surgery reduces short-term complications in lumbar discectomy: an analysis of 4310 patients from the ACS-NSQIP database. Spine (Phila Pa 1976) 38:264-271. https://doi.org/10.1097/BRS. 0b013e3182697b57

16. Vaughan J, Gurusamy KS, Davidson BR (2013) Day-surgery versus overnight stay surgery for laparoscopic cholecystectomy. Cochrane Database Syst Rev. https://doi.org/10.1002/14651858. CD006798.pub4

17. Dravet F, Belloin J, Dupre PF, Francois T, Robard S, Theard JL, Classe JM (2000) Role of outpatient surgery in breast surgery. Prospective feasibility study. Ann Chir 125:668-676. https://doi. org/10.1016/s0003-3944(00)00258-3

18. Ibrahim GM, Bernstein M (2012) Awake craniotomy for supratentorial gliomas: why, when and how? CNS Oncol 1:71-83. https:// doi.org/10.2217/cns.12.1

19. Chacko AG, Thomas SG, Babu KS, Daniel RT, Chacko G, Prabhu K, Cherian V, Korula G (2013) Awake craniotomy and electrophysiological mapping for eloquent area tumours. Clin Neurol Neurosurg 115:329-334. https://doi.org/10.1016/j.clineuro.2012. 10.022

20. Blanshard HJ, Chung F, Manninen PH, Taylor MD, Bernstein M (2001) Awake craniotomy for removal of intracranial tumor: considerations for early discharge. Anesth Analg 92:89-94. https:// doi.org/10.1097/00000539-200101000-00018
21. Purzner T, Purzner J, Massicotte EM, Bernstein M (2011) Outpatient brain tumor surgery and spinal decompression: a prospective study of 1003 patients. Neurosurgery 69:119-127. https://doi.org/ 10.1227/NEU.0b013e318215a270

22. Taylor WA, Thomas NW, Wellings JA, Bell BA (1995) Timing of postoperative intracranial hematoma development and implications for the best use of neurosurgical intensive care. J Neurosurg 82:48-50. https://doi.org/10.3171/jns.1995.82.1.0048

23. Florman JE, Cushing D, Keller LA, Rughani AI (2017) A protocol for postoperative admission of elective craniotomy patients to a non-ICU or step-down setting. J Neurosurg 127:1392-1397. https://doi.org/10.3171/2016.10.Jns16954

24. MacAllister L, Bellanti D, Sakallaris BR (2016) Exploring inpatients' experiences of healing and healing spaces: a mixed methods study. J Patient Exp 3:119-130. https://doi.org/10.1177/23743 73516676182

25. Turel MK, Bernstein M (2016) Is outpatient brain tumor surgery feasible in India? Neurol India 64:886-895. https://doi.org/10. 4103/0028-3886.190227

26. Marigil M, Bernstein M (2018) Outpatient neurosurgery in neurooncology. Neurosurg Focus 44(6): 19

Publisher's Note Springer Nature remains neutral with regard to jurisdictional claims in published maps and institutional affiliations. 